\title{
Pathogenic Microbes Growth Pattern Analysis in Staple Suya Meat Consumption Using the Bayesian Networks
}

\author{
Joe Essien \\ School of Computing and Technology, \\ University of West London, St Mary’s Road, Ealing, London
}

\begin{abstract}
Suya is a spicy meat skewer popular as a food item in the sub-Sahara regions. It is traditionally prepared by the Hausa people of northern Cameroon, Nigeria, Niger, Ghana, and some parts of Sudan. Suya is generally made with skewered beef, ram, chicken and innards such as kidney, liver and tripe. Being a stable meat caviar for people of this region, prepared for consumption and in almost all cases, very unhygienic and sanitary conditions, the beef regalement is exposed massively to microbiome especially Escherichia coli (E. coli). Though most E.coli strains are harmless, some can cause serious food poisoning and severe disease. This paper aims to adopt the generality of Bayesian networks to represent and solve decision problems under uncertainty using influence diagrams from Bayesian taxonomies. It also investigates the application of Bayesian networks principles in making reliable predictions about reoccurrence of microbe growths. Based on the analytical categorisation, the research work builds on extant historical trends which establish referential patterns for microbiome growth in suya meat using Bayesian Networks as a formal model for characterizing and reasoning with uncertain information. Bayesian network models have proven useful in Decision Support Systems (DSS) and in many fields of Artificial Intelligence (AI) including medical domain diagnosis and weather forecasting. This research extends the use of Bayesian networks and analysis in the interdisciplinary studies of information system in microbiology.
\end{abstract}

Keywords: Bayesian Network, Microbiome, Decision Support Systems, Microbiology, Influence taxonomies.

\section{INTRODUCTION}

Bayesian Networks are probabilistic cyclic graphical models that represent finite set of variables and their corresponding dependencies depicted as graphical taxonomies. In medical sciences, Bayesian Network could be used to denote the probabilistic homogeneity between symptom and diseases. Thus Bayesian Networks are usually associated with complex algorithms that realize explicit inferences and learning derivatives. Bayesian network can also relate variables to each other over adjacent time steps enabling the value of a variable to be calculated from the internal regressors and the immediate prior value. This type of networks are referred to as Dynamic Bayesian Network (DBN) and are common in robotics, and have been proven to offer potentials for a wide range of data mining applications such as speech recognition, digital forensic, protein sequencing and bioinformatics. DBN is a generalization of hidden Markov models and Kalman [1] filters and is inferred in this research as it conceptually relates to Probabilistic Boolean Networks [2] and can similarly be used to model dynamical systems at steady-state.

\section{IMPLICATIONS AND MICROBE PATTERNS ANALYSIS}

There are various ailments that meat consumers can contract from endogenously infected meat. These include but are not limited to anthrax, bovine tuberculosis, brucellosis, salmonellosis, listeriosis, trichinosis and taeniasis. The consumption of contaminated brawn as in the case of suya meat in many sub-Saharan communities has been identified as the cause of many health issues. It has been argued that the preparation method for the larder which involves extensive exposure to extreme heat and under hot sun is capable of exterminating any form of extant microbiome growth. However, the presence of infectious agents not exposed to the intensive heat can transmit and retain growths of microorganisms during processing of the meat and after the grilling, re-infest the prepared brawn due to the very unhygienic processes of the entire method. Often, transient microbiome convalesces and breeds over the fructiferous moist dried meat base. Several approaches for examining meat exist such as the Hazard Analysis and Critical Control Point approach (HACCP), [3]. HACCP was developed for the food industry by Pillsbury in the 1960s to make food safe for the then emerging space program. Thereafter, the system has been adopted extensively by many food processors as the approach defines best standard for food safety process management. Other recent approaches for meat testing include use of 
activated lactoferrin (ALF). ALF is a new form of a naturally occurring protein from milk that acts as a powerful deterrent to pathogenic bacteria that may be present on a meat surface. [4]. Other methods for testing meat include sensory evaluation, physical test methods for important parameters such as temperature, acidity $(\mathrm{pH})$, water activity, water binding capacity, physical parameters including light intensity and mechanical testing for texture, chemical and microbiological tests. Using HACCP, meat is meticulously analysed to identify through the processes critical control points (CCP). CCP are steps in the production process where potential biological, physical, and/or chemical hazards can be controlled and may include ensuring the proper cooling of meat to control bacterial growth, or thorough cooking of ready-to-eat products like deli meats to kill bacteria. This approach for instance can identify most high risk parts of the food processing and allows enhancement of processes and allocation resources accordingly. Meat contamination during preparation and processing can be ruminated as a biological prognostication that encompasses alterations in the available substrata such as low molecular compounds during prevalence of a certain microbial affinities with ephemeral spoilage organisms (ESO). ESO are the consequence of factors that dynamically persist or imposed during processing, transportation and storage of meat. When spoilage progresses, most consumers agree that gross discoloration, strong off-odours, and the development of slime would constitute the main qualitative criteria for meat rejection.

However, sensory evaluation, physical scrutiny of parameters and chemical testing by themselves are not sufficient to ensure adequate food safety. The application of Bayesian networks to make estimations on the probability that an incoming occurrence of microorganisms growing or that microorganisms had already grown in the meat evidenced by the historical data and data collected of past occurrence whether true or false can create more insight to the nature of the meat before consumption. Bayesian network can unveil the unique attributes to deal with the different patterns of circumstances in which microorganisms occur in meat products. However, there are questions such as the possibility to create a Bayesian model that fits every type of microorganisms in meat, while for each microorganism different instantiations of patterns apply. Also as a concern is the reliability of the probability model of the Bayesian network in making accurate incisiveness about incoming growth of microorganisms in meat. Because most information available about these probabilities are subjective as well as circumstances of the growth of microorganisms in meat, generic inferences for Bayesian model and probability on the likelihood of growth of microorganisms in the meat product remain interesting subject for more research.

\section{CONCEPTUAL BASIS FOR BAYESIAN NETWORK ANALYSIS}

The aim of the research work of which this paper is supine is to investigate if a Bayesian network could be used to aid in the analysis of incoming or future occurrence of microbiome growth pattern in suya meat. This conceptualization is based on evidence that Bayesian networks are esteemed a rational formal model for representing, and reasoning with uncertain information. Such models have proven useful as decision support systems in several medical situations where information at hand was uncertain and decisions nevertheless needed to be made. They have also been employed in other areas where diagnoses are to be made by domain experts. Consequence to these vicissitude and acknowledgements, the principle of the application of Bayesian network in the analysis of the growth pattern of microorganisms is meshed. In the analysis of growth pattern of microorganisms, Bayesian network represents the probabilistic relationships between diseases and symptoms. Given symptoms, the network can be used to compute the probabilities of the presence of various diseases. The accurate predictive capabilities of Bayesian network to determine whether or not there is the presence of microorganisms in the suya meat products can alert the consumers, the public or community on the potential risks of its consumption. The danger of not knowing the nature of meat products or using Bayesian network to diagnose the disease nature of meat products before consuming them can result in serious health issues. Thus the objective of this research and relevance is to use Bayesian networks to provide a statistical modelling framework that enables analysis and integration of information in its own right as well as integration of a variety of models addressing different aspects of a common problem of potential contaminated meat consumption. Using the Bayesian networks, a probabilistic model will be extrapolated from probability distributions and applied to the laws of probability to predict an anomaly for reasoning and diagnostics, decision making under uncertainty and time series prediction.

\section{HOMEOPATHIC IMPLICATIONS}

Several researches have confirmed that meals consisting of meat, eggs or dairy products also include known carcinogens, bacteria, and other contaminants that can amass in the body for years. Animal products are laden with bacteria, antibiotics, hormones, dioxins, and many other toxins that can cause serious health problems in humans [5]. Research has shown that an extremely high percentage of all the flesh from cattle butchered daily in Nigeria and other sub-Saharan countries is contaminated with high presence of Escherichia coli, campylobacter, listeria, and other opportunistic bacteria that live in the intestinal tracts, flesh, and faeces of animals [6]. Consumption of nutriments contaminated with opportunistic microbiome growth can result in food poisoning, with symptoms ranging from stomach cramps and diarrhoea to organ failure and death. Every year in sub-Saharan Africa and particularly in Nigeria, there are 53 million cases of food poisoning and 6.7 million of these cases lead to complications resulting in fatal consequences. A renown professor of Food Science and Technology, Alfred Ihenkuronye, 
confirmed that in Nigeria alone, more than 200,000 persons die of food poison annually and related the deaths as caused by contaminated foods through improper processing, preservation and service [7]. Many Nutritionist and Dietician at Abuja, Nigeria for instance where there is huge appetite for consumption of suya meat and the Abuja Municipal Area Council (AMAC) Health Department continue in several campaigns to stressed the need for sensitisation for adoption of hygienic processes in the processing of the beef nutriment in order to reduce the incidents of food poison and deaths [8].

To further affirms these prognostication, a major study published in the journal Nature found that a substance found in meat and milk (but not naturally found in the human body) causes humans who eat meat and milk to become more susceptible to dangerous E. coli infections. Thus meat consumption tainted with microbiome growths is often correlated with ingestion of dangerous bacteria which can cause various ailments [9]. Researchers have found that consuming 2 ounces of suya per day - the equivalent of three sticks of the seller's measure - exposes a consumer to 12 to 18 micrograms of inorganic arsenic, the element's most toxic form. Daily exposure to low doses of arsenic can dramatically increase the risk of cancer, dementia, neurological problems, and other ailments in humans [10].

Consumer knowledge of food safety and awareness of hazards in food is important in reducing the prevalence of foodborne diseases. Microbiological assessments of foods in Nigeria particularly street vended foods have been demonstrated to be problematic [11]. The prevalence of foodborne pathogens in foods consumed in Sub-Saharan Africa and particularly Nigeria is alarming and of public health concern [12], [13]. Suya is a popular Nigerian roasted meat product prepared, processed and sold under unhygienic conditions as with majority of other ready to eat street vended foods [14], [15], [16], [17], [18]. Dioxins are chemicals that are released into the environment when substances are burned, and they accumulate in animals' flesh. According to leading scientists and the Environmental Protection Agency (EPA), nearly 95 Percent of our dioxin exposure comes in the concentrated form of meat, fish, and dairy products, because when animal products are eaten, the dioxin that animals have built up in their bodies is absorbed into the body [19]. Dioxin is a powerful hormone-disrupting group of chemicals that binds to a cell and modifies its functioning, potentially causing a wide range of effects, including cancer, depressed immune response, nervous system disorders, miscarriages, and birth deformities. Suya consumption has been reported to result in these sporadic outbreaks of foodborne diseases [14], [20]. Moreover, suya is documented to have high level of Polynuclear Aromatic Hydrocarbons (PAH) [21], and indiscriminate consumption of suya spices has hematologic effects [22] with potential health hazards. Consumer understanding and perceptions of food safety are considered to form the basis of a heuristic framework that guides decisions about behaviour. There is increase desire to consume minimally cooked or raw foods worldwide thereby minimizing the consumer risk perception of hazards in foods. According to Liu, Yuan et al. [23], one strategy to reducing foodborne illness involves implementing food-safety education programs for both consumers and food-handlers. In order to properly design these educational programs, basic and fundamental information is needed as to the eating habits, personal hygiene and food safety knowledge of consumers. Therefore, the goal of this study is to identify some risky behaviour consumers engage in and to provide information on public awareness of food safety and foodborne diseases among consumers meat, especially suya meat.

\section{BAYESIAN NETWORK MODEL AND MICROBES ANALYSIS}

The earliest work on medical expert systems was based on Bayesian approach [24] where Bayesian networks were applied to manage infectious diseases in intensive care units. Dynamic Bayesian networks (DBNs), a temporal extension of Bayesian networks, found their applications in medicine where selected applications included DBN for management of patients suffering from a carcinoid tumour, or NasoNet, a system for diagnosis and prognosis of nasopharyngeal cancer. DBNs have also been used in cellular systems or for modeling dynamics of organ failure in patients in intensive care units.

In this disposition, Bayesian network models are used to produce posterior probability distributions over hypothesis that it is possible to make reliable predictions about reoccurrence of microbes' growths in suya meat. In case of a diagnostic network, the output of a model is percolated as an assignment of posterior probabilities to various disorders. In order to make a diagnostic decision, the probabilities of rival hypotheses (in the idealized case, the joint probability distribution over all disorders) are determined. This allows for weighting the utility of correct diagnosis against the disutility of incorrect diagnosis. Applying the focus of reasoning as a differential diagnosis, it is possible to observe how the posterior in question compares to the posteriors of competing disorders.

As this research is not focused on carrying out an idiopathic microbiological analysis as justice in form of research works have already been done on this premise [15], [20], [25], [26], [27], this research acquires its ferocity by exploiting the existing large and varied database of secondary data obtained for this works to advance the novel approach of applying predictive microbe analysis using Bayesian Networks. The data are extrapolated into the Bayesian network models that describe the kind of analysis desired for deducing inferences and reliable predictions about reoccurrence of microbe growths in suya meat. Using the Bayesian network approach ensures that the probabilities that result at each stage of knowledge build-up are mathematically and scientifically 
thorough. In other words, given the data obtained about the meat, and then based on the best mathematical and statistical knowledge, the Bayesian network (Bayes Nets or BN) can predict what can be legitimately expected by application of the Bayes Rule;

$$
\begin{aligned}
& p(B \mid A)=p(A \mid B) x p(B) / p(A) \\
& \text { Where } \mathrm{p}(\mathrm{A})^{\prime} \text { is "the probability of } \mathrm{A} ", \\
& \text { and } \\
& \mathrm{p}(\mathrm{A} \mid \mathrm{B}) \text { ' is "the probability of A given that B has occurred". }
\end{aligned}
$$

The presence or growth of microbe patterns in meat is identified by any one of the dependencies or symptoms perceptible in the meat specimens. That is, the probability of an acerbic taste occurring given that there is a variance in odour or the probability of microbe growth pattern development in the meat given that there is change in odour of the meat equals the probability of the pathogenic microbes growth pattern multiplied by the probability of change in odour of the meat given that there is microorganism in the meat divided by the probability of change in the odour of the meat.

Mathematically, this can be represented as:

$$
P(M \mid C)=P(M) * P(C \mid M) / P(C)
$$

$$
\begin{aligned}
& \text { Where } \\
& M=\text { Microorganism and } C=\text { Change in odour. } \\
& P(C) \text { - denotes the probability of } C \text {. } \\
& P(C, M) \text { denotes the joint probability of } C \text { and } M . \\
& P(C \mid M) \text { denotes the conditional probability of } C \text { given } M \text {. }
\end{aligned}
$$

Following the $\mathrm{BN}$ definition: $\mathrm{P} r(\mathrm{~A}, \mathrm{~B}, \mathrm{C})=\mathrm{P} r(\mathrm{~A}) \mathrm{P} r(\mathrm{~B} \mid \mathrm{A}) \mathrm{P} r(\mathrm{C} \mid \mathrm{B})$. To decide on conditional independence between $\mathrm{A}$ and $\mathrm{C}$, $\mathrm{P}$ $\mathrm{r}(\mathrm{A}, \mathrm{C} \mid \mathrm{B})$ can be expressed and factorized. It follows from both conditional independence and $\mathrm{BN}$ definition: $\mathrm{P} r(\mathrm{~A}, \mathrm{C} \mid \mathrm{B})=\mathrm{P} r(\mathrm{~A}$,

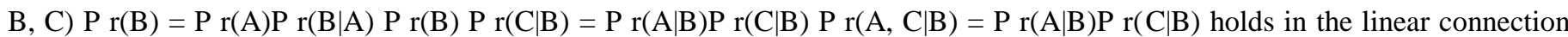
and $\mathrm{A} \perp \perp \mathrm{C} \mid \mathrm{B}$ also holds. Applying this mathematical theorem on the sample statistics obtained for this analysis (Table 1), this can be expressed as several probabilities of the existence of growth patterns of microbes in the suya meat samples.

Table 1: Prevalence of Microbes in Suya Samples (Ogbu et al., 2016)

\begin{tabular}{|l|c|c|}
\hline \multicolumn{1}{|c|}{ MICROBES } & SUYA SAMPLES & PREVALENCE (\%) \\
\hline Salmonella species & 13 & $18.84 \%$ \\
\hline Klebsiella species & 6 & $8.70 \%$ \\
\hline Escherichia coli & 9 & $13.04 \%$ \\
\hline Enterobacter species & 7 & $10.14 \%$ \\
\hline Staphylococcus species & 5 & $7.25 \%$ \\
\hline Streptococcus Species & 4 & $5.80 \%$ \\
\hline Bacillus species & 3 & $4.35 \%$ \\
\hline Shigella species & 14 & $20.29 \%$ \\
\hline Serratia species & 8 & $11.59 \%$ \\
\hline Total & 69 & $100 \%$ \\
\hline
\end{tabular}

In order to be certain about the growth of Pathogenic Microbes Pattern, several other characteristics can be identified such as odour, change of colour and fungi growth [20],[22] as represented in sample data in Tables 2,3,4,5, 6 and 7.

Table 2: $\mathbf{P}$ (Sensory characteristics $\mid \Delta$ in Odour) 
International Journal of Advances in Scientific Research and Engineering (ijasre), Vol 5 (4), April-2019

\begin{tabular}{|c|l|c|c|}
\hline Souring & $\begin{array}{l}\text { Fat } \\
\text { decompositio } \\
\text { n }\end{array}$ & $\begin{array}{l}\Delta \text { in } \\
\text { Odour }= \\
\text { True }\end{array}$ & $\begin{array}{l}\Delta \text { in odour } \\
\text { = False }\end{array}$ \\
\hline True & True & 0.9 & 0.1 \\
\hline True & False & 0.8 & 0.2 \\
\hline False & True & 0.7 & 0.3 \\
\hline False & False & 0.1 & 0.9 \\
\hline
\end{tabular}

Table 3: P (Souring | Has Foul Odour, $\Delta$ in Odour)

\begin{tabular}{|l|c|c|c|}
\hline Has Foul odour & $\begin{array}{c}\text { Change in } \\
\text { odour }\end{array}$ & $\begin{array}{c}\text { Souring } \\
=\text { True }\end{array}$ & $\begin{array}{c}\text { Souring } \\
=\text { False }\end{array}$ \\
\hline True & True & 0.9 & 0.1 \\
\hline True & False & 0.8 & 0.2 \\
\hline False & True & 0.3 & 0.7 \\
\hline False & False & 0.9 & 0.1 \\
\hline
\end{tabular}

Table 4: P (Has Foul odour | Gas Production)

\begin{tabular}{|l|c|c|}
\hline $\begin{array}{l}\text { Gas } \\
\text { Production }\end{array}$ & $\begin{array}{c}\text { Has Foul Odour } \\
=\text { True }\end{array}$ & $\begin{array}{c}\text { Has Foul Odour } \\
\text { False }\end{array}$ \\
\hline True & 0.6 & 0.4 \\
\hline False & 0.3 & 0.7 \\
\hline
\end{tabular}

Table 5: P (Has Discoloration | Gas Production)

\begin{tabular}{|l|c|c|}
\hline $\begin{array}{l}\text { Gas } \\
\text { Production }\end{array}$ & $\begin{array}{c}\text { Has Discoloration } \\
\text { = True }\end{array}$ & $\begin{array}{c}\text { Has Discoloration } \\
\text { = False }\end{array}$ \\
\hline True & 0.1 & 0.9 \\
\hline False & 0.99 & 0.01 \\
\hline
\end{tabular}

Table 6:P (Microorganisms in meat | Discoloration)

\begin{tabular}{|l|c|c|}
\hline $\begin{array}{l}\text { Has } \\
\text { Discoloration }\end{array}$ & $\begin{array}{c}\text { Microorganisms } \\
\text { in meat = True }\end{array}$ & $\begin{array}{c}\text { Microorganisms } \\
\text { in meat = False }\end{array}$ \\
\hline True & 0.99 & 0.01 \\
\hline False & 0.01 & 0.99 \\
\hline
\end{tabular}

Table 7:P(Has Surface Slime 1 Microbes in suya meat)

\begin{tabular}{|l|c|c|}
\hline Microbes in meat & $\begin{array}{c}\text { Has Surface } \\
\text { Slime = True }\end{array}$ & $\begin{array}{c}\text { Has Surface } \\
\text { Slime = False }\end{array}$ \\
\hline True & 0.95 & 0.05 \\
\hline False & 0.01 & 0.99 \\
\hline
\end{tabular}

Bayes rule can be extended to multiple variables with multiple states. Though such equations are far more complex to write out and problematic to compute manually, it is easy for analysis and computation with the Bayes Net. 


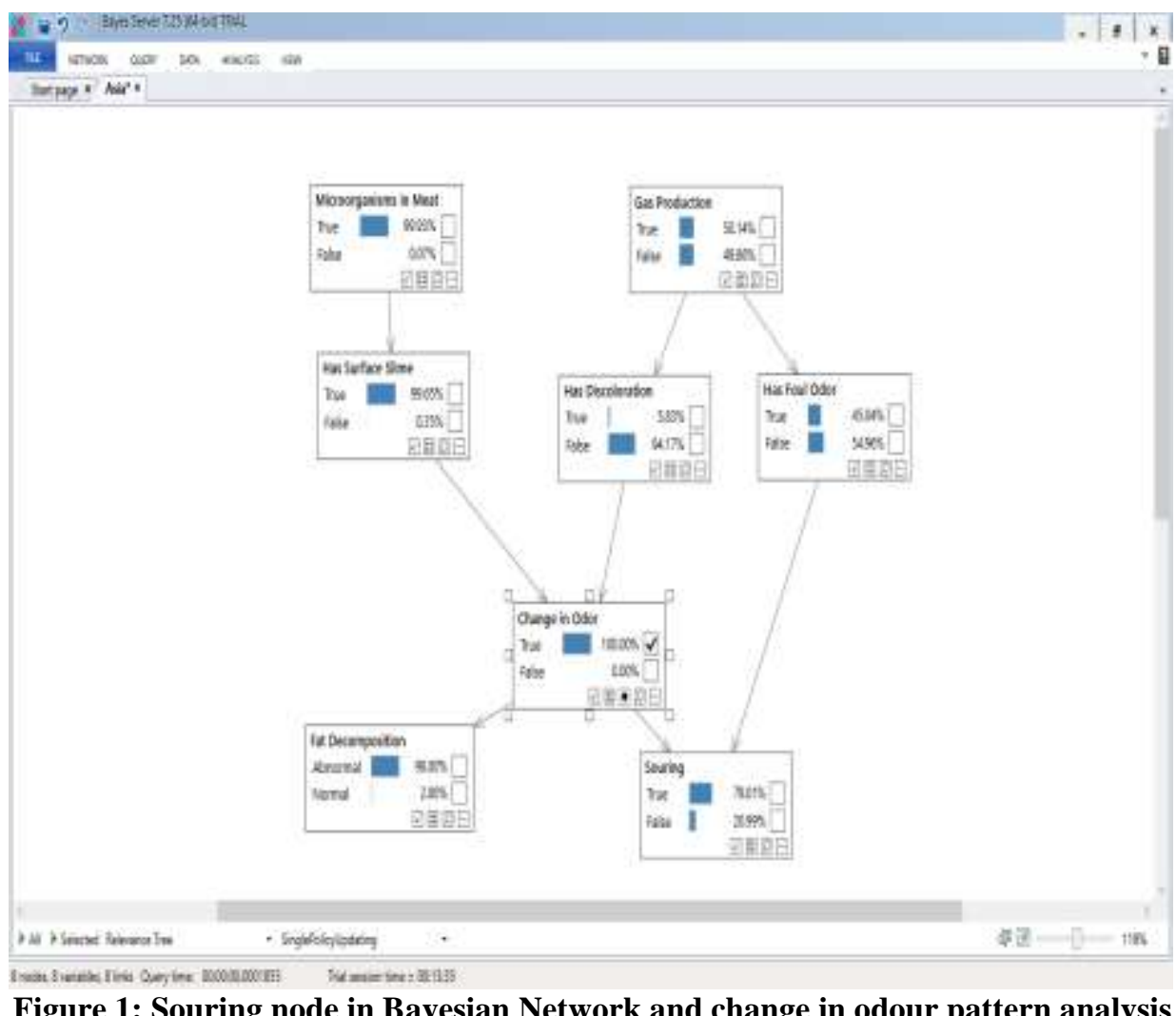

In Figure 1, the ticked box in the node of change in odour shows that the change in odour of the meat is responsible for the surface slime, souring, fat decomposition abnormality of the meat and the presence of microorganisms in the meat. This means the presence of a single symptom can lead to a multitude of several other symptoms in the meat. The arithmetic averages are extrapolated where their meaning added clarification to observations or where there is need to determine the overall trend of a data set or providing a rapid snapshot of the data. The standard deviation is used to measure the spread of data around the mean. A high standard deviation connoted wider spread of data from the mean, while a low standard deviation signalled that more data align with the mean. In a portfolio of data analysis methods adopted here, the standard deviation is useful for quickly determining dispersion of data points. Regression models were also decrypted to show relationships between dependent and explanatory variables and charted as scatterplot where necessary. The regression line also designates whether those relationships are strong or weak and facilitated the determination of trends over time. It also portrays a flexible extension of maximum likelihood thus providing an efficient method to fit the statistical model.

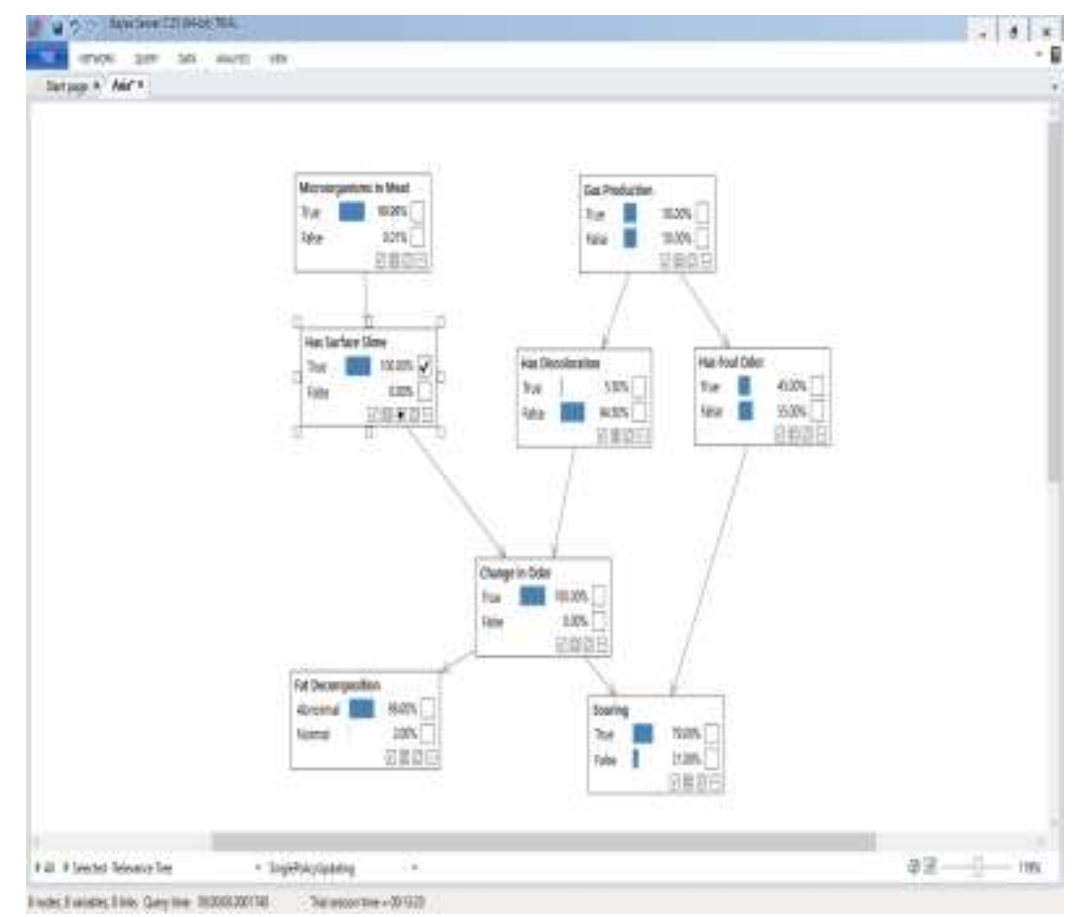

Figure 2: Souring node in Bayesian Network and change in Surface Slime pattern analysis 
A sample run of the Bayesian application for analysing the growth pattern of microbes in meat infers an increase in surface slime in the meat when increased chances of the presence of microorganisms in the meat and other microbial variables in the network are simulated.

The two top nodes in Figure 3 are for predispositions which influence the likelihood of the pathogenic microbes' pattern growth. Those growth patterns appear in the row below them. At the bottom are predictions about reoccurrence. To a large degree, the links of the network correspond to causation. This is a common structure for diagnostic networks: predisposition nodes at the top, with links to nodes representing internal conditions and failure states, which in turn have links to nodes for observables.

Often there are many layers of nodes representing internal conditions, with links between them representing their complex interrelationships.

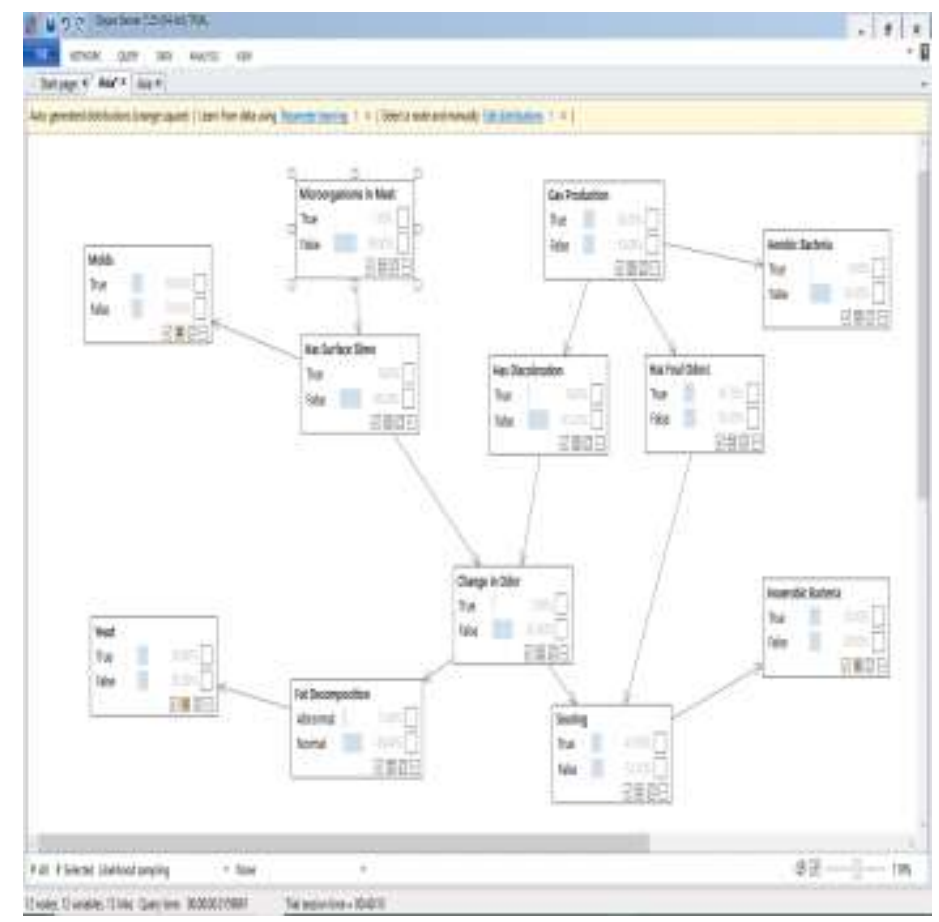

Figure 3: Depiction of effect of discoloration on related nodes and symptoms in the Bayes Net.

Bayesian data analysis carried out in these exposition apples the probability to represent uncertainty in all parts of the statistical model thus offering a flexible extension of maximum likelihood and an information-efficient method to fit a statistical model for computing complex and intensive analysis.

\section{SUMMARY AND CONCLUSION}

Interdisciplinary studies conducted in the field of computing, artificial intelligence and microbiome have provided a greater insight into the relationship between meat-handling techniques prior to garnering and the process of meat preparation for consumption using hygienic methodologies. Improved ways of meat storage and processing have continued to be a subject for circumspection. These include practices while butchering, especially in sub-Saharan countries where there are little or no infrastructure to support sanitised management of the meat, improvements to refrigeration and storage, transportation, aging, and improving the end product thus reduce sickness and health issues. The storage life of fresh meats is largely determined by the number and nature of the microorganisms that grow on or in the animal tissue. There are diverse sources of these organisms and measure to minimize their numbers on the product must be taken into consideration, the flora associated with the animal and methods by which the animal is processed. Bayesian data analysis provides the means to extrapolate and apply probability to represent uncertainty in statistical model for meat analysis. It is also a flexible extension of maximum likelihood and potentially the most information-efficient method to fit a statistical model though it could be hypothetically computationally intensive. With Bayesian data analysis it is possible to anatomise unknowns from predetermined parameters based on data, generative models and priors. Thus the Bayesian networks formalism can be applied to build or analysed a system that can assist in the diagnosis concerning the validity of microbe growth patterns in meat using historical data of earlier samples of the growth of microorganisms which is the rational for use of secondary data for this analysis to make decisions about future incoming growth of microbe patterns. One advantage of Bayesian networks is that it is intuitively easier for a human to understand a sparse set of direct dependencies and local distributions than complete joint distributions. 


\section{REFERENCES}

1. Kim, Chang-Jin. "Dynamic linear models with Markov-switching." Journal of Econometrics 60.1-2 (1994): 1-22.

2. Lähdesmäki, Harri, et al. "Relationships between probabilistic Boolean networks and dynamic Bayesian networks as models of gene regulatory networks." Signal processing 86.4 (2006): 814-834.

3. Gilling, Samantha J., et al. "Successful hazard analysis critical control point implementation in the United Kingdom: understanding the barriers through the use of a behavioral adherence model." Journal of Food Protection 64.5 (2001): 710-715.

4. Naidu, A. Satyanarayan. "Activated Lactoferrin- A New Approach to Meat Safety." Food Technology 56.3 (2002): 4046.

5. Kinlaw, William B., et al. "Fatty acids and breast cancer: make them on site or have them delivered." Journal of cellular physiology 231.10 (2016): 2128-2141.

6. $\quad$ Bintsis, Thomas. "Microbial pollution and food safety." AIMS Microbiology 4.3 (2018): 377-396.

7. Ajayi, Olasunmbo Abolanle, and Taiwo Salaudeen. "Consumer food safety awareness and knowledge in Nigeria." Internet Journal of Food Safety 16 (2014): 17-24.

8. Alum, Eucharia Akanele, S. M. O. C. Urom, and Chukwu Mary Ahudie Ben. "Microbiological contamination of food: the mechanisms, impacts and prevention." Int. J. Sci. Technol. Res 5.3 (2016): 65-78.

9. Balch, James F. The Super Anti-Oxidants: Why They Will Change the Face of Healthcare in the 21st Century. M. Evans, 1999.

10. Abdul, Khaja Shameem Mohammed, et al. "Arsenic and human health effects: A review." Environmental toxicology and pharmacology 40.3 (2015): 828-846.

11. Ajayi, Olasunmbo A., and Jacob O. Oluwoye. "Sustainable Street vended foods and food safety: a conceptual framework." International Journal of Food Safety, Nutrition and Public Health 5.3-4 (2015): 195-216.

12. Ehiri, John E., et al. "Critical control points of complementary food preparation and handling in eastern Nigeria." Bulletin of the World Health Organization 79 (2001): 423-433.

13. Uzeh, Roseline E., Regina E. Ohenhen, and Ayodeji K. Rojugbokan. "Microbiological and nutritional qualities of dairy products: Nono and Wara." Nature and science 4.3 (2006): 37-40.

14. Ogbonna, Innocent Okonkwo, et al. "Microbiological Safety and Proximate Composition of (Suya) Stored at Ambient Temperature for Six Hours from Maiduguri, Northern Nigeria." Internet J. Food Saf 14 (2012): 11-16.

15. Uzeh, Roseline E., Regina E. Ohenhen, and Ayodeji K. Rojugbokan. "Microbiological and nutritional qualities of dairy products: Nono and Wara." Nature and science 4.3 (2006): 37-40.

16. Ologhobo, A. D., et al. "Safety of street vended meat products-chicken and beef suya." African Journal of biotechnology 9.26 (2010): 4091-4095.

17. Madueke, S. N., S. Awe, and A. I. Jonah. "Microbiological analysis of street foods along Lokoja-Abuja express way, Lokoja." American Journal of Research Communication 2.1 (2014): 196-211.

18. Ajayi, Olasunmbo A., and Jacob O. Oluwoye. "Sustainable Street vended foods and food safety: a conceptual framework." International Journal of Food Safety, Nutrition and Public Health 5.3-4 (2015): 195-216.

19. Van den Berg, Martin, et al. "The 2005 World Health Organization reevaluation of human and mammalian toxic equivalency factors for dioxins and dioxin-like compounds." Toxicological sciences 93.2 (2006): 223-241.

20. Olayinka, Edema Mojisola, Osho Adetokunbo Temitope, and Diala Chukwunonye Innocent. "Evaluation of microbial hazards associated with the processing of Suya (a grilled meat product)." Scientific Research and Essays 3.12 (2008): 621-626.

21. Ogbuagu, Dike Henry, and Adedolapo Abeke Ayoade. "Presence and levels of common polynuclear aromatic hydrocarbons (PAHs) in staple foods of Nigerians." Food and Public Health 2.1 (2012): 50-54.

22. Adeyeye, Samuel Ayofemi Olalekan. "Effect of Processing Methods on Quality and Safety of Suya, a West African Grilled Meat." Journal of culinary science \& technology 15.2 (2017): 158-170.

23. Liu, Yuan, Xing-lian Xu, and Guang-hong Zhou. "Changes in taste compounds of duck during processing." Food chemistry102.1 (2007): 22-26.

24. Horvitz, Eric J., John S. Breese, and Max Henrion. "Decision theory in expert systems and artificial intelligence." International journal of approximate reasoning 2.3 (1988): 247-302.

25. Ologhobo, A. D., et al. "Safety of street vended meat products-chicken and beef suya." African Journal of biotechnology 9.26 (2010): 4091-4095.

26. Inyang, C. U., M. A. Igyor, and E. N. Uma. "Bacterial quality of a smoked meat product ("Suya")." Nigerian Food Journal 23.1 (2005): 239-242.

27. Chukwura, E. I., and C. N. Mojekwu. "A Short Communication: Prevalence of Microbial Contaminants on "Suya meat" sold in Awka Urban." Journal of Tropical Microbiology and Biotechnology 1.1 (2002): 89-91. 